UDC 533.9

\author{
${ }^{1}$ Arkhipov Yu.V., ${ }^{1 *}$ Ashikbayeva A.B., ${ }^{1}$ Askaruly A., \\ ${ }^{1}$ Yerkinbayev N., ${ }^{1}$ Davletov A.E., and ${ }^{2}$ Tkachenko I.M. \\ ${ }^{1}$ IETP, Faculty of Physics and Technology, al-Farabi Kazakh National University, Almaty, Kazakhstan, \\ ${ }^{2}$ Departamento de Matemática Aplicada, Universidad Politécnica de Valencia, Valencia, Spain \\ e-mail: assel_02@mail.ru
}

\title{
Stopping power and straggling in two-component plasmas
}

\begin{abstract}
Stopping power $S$ is one of the tools of plasma diagnostics. Particularly, the "minus first" (projectile) velocity power moment of the plasma stopping power or the stopping high-velocity asymptotic form. Usually, the interaction of target electrons with the plasma ions is neglected. Nevertheless, the target plasma electron-ion static structure factor influenceson the plasma polarizational stopping power. This effect hasbeen studied using the Feynman-like form for the plasma loss function $L(k, \omega)=-\operatorname{Im} \varepsilon^{-1}(k, \omega) / \omega$ stemming from the canonical solution of themoment problem of reconstruction of the system inverse dielectric function $\varepsilon^{-1}(k, \omega)$. The same approach is employed in the present work tostudy the two-component non-ideal degenerate plasma straggling and itshigh-velocity asymptotic form. At the same time, the non-canonical solution of the moment problem is used to calculate the straggling at anyprojectile velocity. Note that in hot equilibrium plasmas $\left(T>>T_{F}\right)$ the straggling is just $\Omega^{2}=\left(2 k_{B} T\right) S$.
\end{abstract}

Key words: two-component plasmas, stopping power, straggling, loss function

\section{Introduction}

\section{Plasma stopping power}

The laser-induced or heavy-ion-induced implosion of fusion fuel pellets startsfrom normal solid-state conditions and leads to the extreme conditions of inertially confined plasmas in a distinct domain of warm dense matter (WDM) ofhigh densities and temperatures. Usually, the plasma stopping power is definedas the magnitude of the mean energy loss per unit path length: $S=-\Delta E / \Delta x$. Clearly, the treatment of the stopping power of these manmade plasmas requirea quantummechanical formulation in all ranges of plasma coupling and degeneracy. The quantum-mechanical description of the energy loss in a way that canbe immediately applied to plasmas under various conditions was obtained anddiscussed in detail in [1, $2,3]$ to name a few. The polarizational contribution to the stopping power $S$ of an electron onecomponent plasma relates it to the system loss function $L(k, \omega)=-\operatorname{Im}^{-1}(k, \omega) / \omega$, where $\varepsilon^{-1}(k, \omega)$ is the plasma inverse dielectric function:

$$
S(v)=\frac{2\left(Z_{p} e\right)^{2}}{\pi v^{2}} \int_{0}^{\infty} \frac{d k}{k} \int_{0}^{k v} \omega^{2} L(k, \omega) d \omega,
$$

where $v, Z_{p} e$ are the projectile velocity and charge. A contemporary discussion of the topic can be found in [4].

\section{Method of moments}

The stopping power is also an important tool of plasma diagnostics. Particularly, the "minus first" (projectile) velocity power moment of the stopping power [5] or the stopping high-velocity asymptotic form [6],

$$
S(v \rightarrow \infty)=\left(\frac{Z_{p} e \omega_{p}}{v}\right)^{2} \operatorname{Ln} \frac{2 m_{e} v^{2}}{\hbar \omega_{p}},
$$

where $\omega_{p}$ is the plasma frequency. Usually, the interaction of target electrons with the plasma ions is neglected in the stopping power. Nevertheless, the target plasma electron-ion static structure factor influences the plasma polarizational stopping power. This effect has been studied using the Feynman-like form for the plasma loss function [7]: 


$$
\begin{gathered}
\frac{L(k, \omega)}{\pi C_{0}(k)}=\frac{\omega_{2}^{2}(k)-\omega_{1}^{2}(k)}{\omega_{2}^{2}(k)} \delta(\omega)+ \\
+\frac{\omega_{1}^{2}(k)}{2 \omega_{2}^{2}(k)}\left[\delta\left(\omega-\omega_{2}(k)\right)+\delta\left(\omega+\omega_{2}(k)\right)\right],
\end{gathered}
$$

stemming from the canonical solution of the moment problem of reconstruction of the system inverse dielectric function $\varepsilon^{-1}(k, \omega)$ [8]. Here, the characteristic frequencies

$$
\omega_{j}(k)=\sqrt{\frac{C_{2 j}(k)}{C_{2 j-2}(k)}}, \quad j=1,2,
$$

and

$$
C_{v}(k)=\pi^{-1} \int_{-\infty}^{\infty} \omega^{v} L(k, \omega) d \omega, \quad v=0,1, \ldots
$$

are the power frequency moments of the loss function. Due to the parity of the latter, all oddorder frequency moments vanish. The even-order frequency moments are determined by the static characteristics of the system. After a straightforward calculation one obtains:

$$
\begin{aligned}
& C_{0}(k)=\left(1-\varepsilon^{-1}(k, 0)\right), \quad C_{2}(k)=\omega_{p}^{2} \\
& C_{4}(k)=\omega_{p}^{4}(1+K(k)+U(k)+H),
\end{aligned}
$$

with $K(k)=\left(\left\langle v_{e}^{2}\right\rangle k^{2}+\hbar^{2} k^{4} /(2 m)^{2}\right) / \omega_{p}^{2},\left\langle v_{e}^{2}\right\rangle$

being the average squared characteristic velocity of the plasma electrons. The last two terms in the fourthmoment stem from the interaction contribution to the system Hamiltonian and can be expressed in terms of the partial structure factors $S_{a b}(k), a, b=e, i$ :

$$
\begin{gathered}
U(k)=\left(2 \pi^{2} n_{e}\right)^{-1} \int_{0}^{\infty} p^{2}\left(S_{e e}(p)-1\right) f(p, k) d p, \\
H=\left(6 \pi^{2} n_{e}\right)^{-1} \int_{0}^{\infty} p^{2} S_{e i}(p) d p,
\end{gathered}
$$

where we have introduced

$$
\begin{gathered}
f(p, k)=5 / 12-p^{2} /\left(4 k^{2}\right)+ \\
+\left(k^{2}-p^{2}\right)^{2} \operatorname{Ln}|(p+k) /(p-k)| /\left(8 p k^{3}\right),
\end{gathered}
$$

and plasma is modelled as a hydrogen-like system with $n_{e}=Z n_{i}$. The Nevanlinnaformula of the theory of moments expresses the dielectric function, which satisfies the known sum rules $\left\{C_{2 v}\right\}_{v=0}^{2}$,

$$
\varepsilon^{-1}(k, z)=1+\frac{\omega_{p}^{2}(z+q)}{z\left(z^{2}-\omega_{2}^{2}\right)+q\left(z^{2}-\omega_{1}^{2}\right)},
$$

in terms of a function $q=q(k, z)$, which is analytic in the upper complex half plane $\operatorname{Im} z>0$ and possesses there a positive imaginary part. It must also satisfy the limiting condition: $(q(k, z) / z) \rightarrow 0$ as $z \rightarrow \infty$ for $\operatorname{Im} z>0$. In an electron liquid, the Nevanlinna parameter function plays the role of the dynamic local-field correction (LFC) $G(k, \omega)$. In particular, the Ichimaruvisco-elastic model expression for $G(k, \omega)$ is equivalent to the Nevanlinna function approximated as $i / \tau_{m}, \tau_{m}$ being the effective relaxation time of the Ichimaru model [9]. In a multi-component system, the Nevanlinna parameter function stands for the species' dynamic LFC's. In general, we do not have enough phenomenological conditions to determine the function $q(k, \omega)$ which would lead to the exact expression for the loss function. One might benefit [8] from the Perel' - Eliashberg [10] expression for the high-frequency asymptotic form of the imaginary part of the dielectric function of the system considered here,

$$
\operatorname{Im} \varepsilon\left(k, \omega>>(\beta \hbar)^{-1}\right) \approx \frac{4 \pi A_{0}}{\omega^{9 / 2}},
$$

where

$$
A_{0}=\frac{2^{5 / 2} \pi}{3} n_{e} n_{i} \frac{Z^{2} e^{6}}{\left(\hbar m_{e}\right)^{3 / 2}} .
$$

This result also implies that higher even-order frequency moments, $C_{2 l}(k), l \geq 3$, diverge. The model (3) corresponds to the limiting case with $q(k, \omega)=i 0^{+}$. It was shown in [7] using (3) that in a hydrogen-like two-component plasma, (2) is substituted by

$$
S_{T C P}(v \rightarrow \infty)=\left(\frac{Z_{p} e \omega_{p}}{v}\right)^{2} \operatorname{Ln} \frac{2 m_{e} v^{2}}{\hbar \omega_{p} \sqrt{1+H}} .
$$




\section{Energy-loss straggling}

The second quantity of interest to characterize the slowing down process isthe energy-loss straggling $\Omega^{2}(v)$, which describes the statistical fluctuations ofthe energy loss of the particle and is defined [2] as the square of the standarddeviation of the energy-loss distribution per unit path length, i.e.,

$$
\Omega^{2}(v)=\frac{\left\langle(\Delta E)^{2}\right\rangle-\langle\Delta E\rangle^{2}}{\Delta x} .
$$

The polarizational contribution to the straggling is also defined by the systemloss function:

$$
\Omega^{2}(v)=\frac{2\left(Z_{p} e\right)^{2} \hbar}{\pi v^{2}} \int_{0}^{\infty} \frac{d k}{k} \int_{0}^{k v} \omega^{3} L(k, \omega) \operatorname{coth} \frac{\beta \hbar \omega}{2} d \omega,
$$

where $\beta^{-1}$ is the target plasma temperature. Now, the same procedure whichled to (6) for the straggling asymptotic form give the following expression:

$$
\Omega_{T C P}^{2}(v \rightarrow \infty)=\frac{\left(Z_{p} e \omega_{p}\right)^{2} \hbar}{v^{2}} \int_{\omega_{p} \sqrt{1+H / v}}^{2 m v / \hbar} \omega_{2}(k) \operatorname{coth}\left(\frac{\beta \hbar \omega_{2}(k)}{2}\right) \frac{d k}{k} .
$$

It is well known that in classical systems $(\beta \rightarrow 0), \Omega^{2}(v)=2 S(v) / \beta$. Similarly, when $\beta \rightarrow 0$ and $\hbar \rightarrow 0$,

$$
\Omega^{2}(v \rightarrow \infty)=\frac{2 S(v \rightarrow \infty)}{\beta}
$$

We have calculated both the stopping and straggling and their asymptoticforms. The NPF was chosen to satisfy the Perel'-Eliashberg asymptotic form(5):

$$
Q_{2}(k, z)=\frac{4 \pi A_{0} \sqrt{z}(1+i)}{\omega_{p}^{2}\left(\omega_{2}^{2}(k)-\omega_{1}^{2}(k)\right)} .
$$

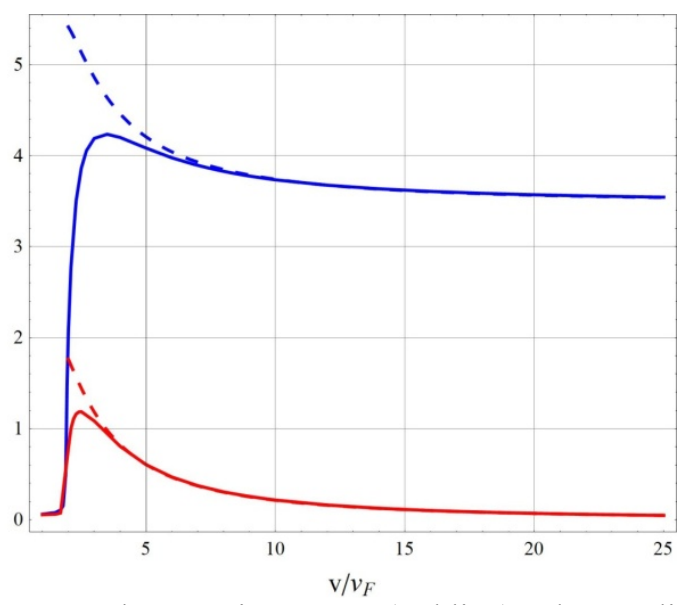

Figure 1 - The stopping power (red line)and straggling (blue line), and their asymptotic forms (dashed lines ofrespective colors) for $\Gamma=1.077$.

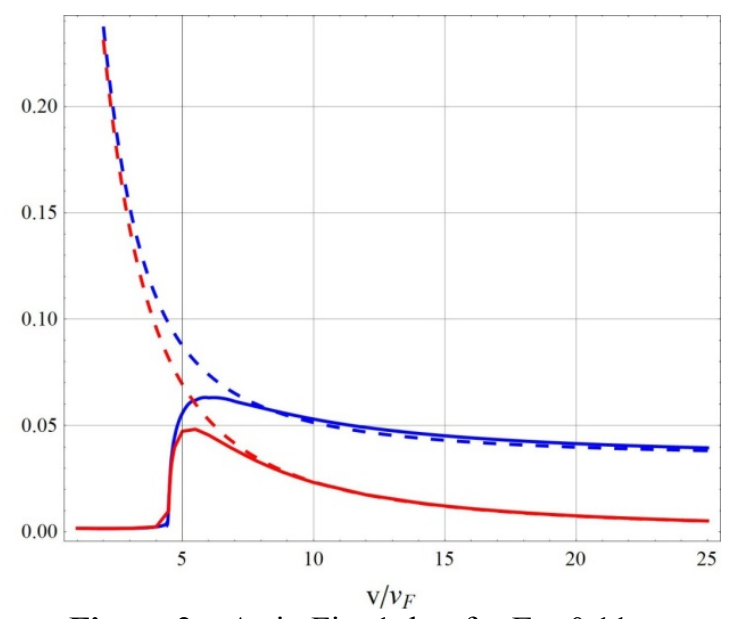

Figure 2 - As in Fig. 1, but for $\Gamma=0.11$

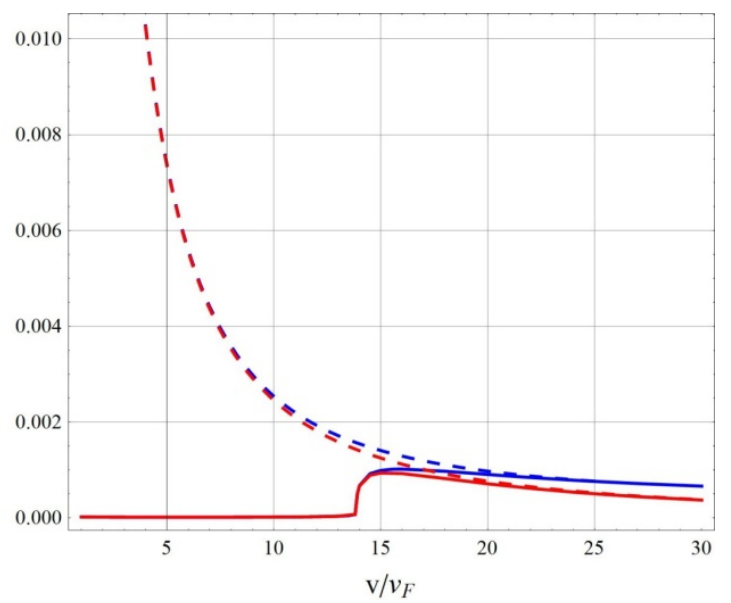

Figure 3 - As in Fig. 1, but for $\Gamma=0.011$ : 


\section{Results and conclusion}

The target plasma static structure factors were calculated in the hyper-netted approximation. The results are presented in Figs. 1-3, where the stopping power and straggling, and their asymptotic forms for very fast projectiles are displayed for $r_{s}=2.5256$ and two different values of the coupling parameter $\Gamma=\beta e \sqrt[3]{4 \pi n_{e} / 3}, r_{s} \quad$ being the Brueckner parameter. These numericaldata tentatively confirm the above analytic results, which might serve for the WDM diagnostics.

\section{References}

1. Lindhard J. On the Properties of a Gas of Charged Particles //Mat.-Fys. Medd. - K. Dan. Vidensk. Selsk. - 1954. - Vol. 28. - No. 8. - P. 1-58.

2. Arista N., Brandt W.Energy loss and straggling of charged particles in plasmas of all degeneracies // Phys. Rev. A. - 1981. - Vol. 23. - P. 1898.

3. Ortner J., Tkachenko I.M. Stopping power of strongly coupled electronic plasmas: Sum rules and asymptotic forms // Phys. Rev E. - 2001. - Vol. 63. - P. 026403.
4. Arkhipov Yu.V. et al. Dielectric function of dense plasmas, their stopping power, and sum rules // Phys. Rev. E. - 2014. - Vol. 90. - P. 053102; 2015. - Vol. 91. - P. 019903.

5. ArkhipovYu.V.et al. The Plasma Stopping Power Velocity Moment Diagnostics // Contr. Plasma Phys. - 2014. - Vol. 54. - P. 308-313.

6. Bethe H. Bethe, H. Zur Theorie des Durchgangsschneller Korpuskularstrahlendurch Materie // Ann. Phys. (Leipzig). - 1930. - Vol. 5. P. 325-400; Larkin A.I. Passage of particles through a plasma //Sov. Phys. JETP. -1960. - Vol. 10. -P. 186-191.

7. Ballester D., Tkachenko I.M. Fast-Projectile Stopping Power of Quantal Multicomponent Strongly Coupled Plasmas // Phys. Rev. Lett. 2008. - Vol. 101. -P. 075002; J. Phys. A: Math. Theor. - 2009. - Vol. 42. - P. 214035.

8. Tkachenko I.M., Arkhipov Yu.V., Askaruly A. The Method of Moments and its Applications in Plasma Physics. - Germany: LAMBERT Academic Publishing, 2012. - 132 p.

9. Ichimaru S. Statistical plasma physics, Vol. 2: Condensed plasmas. - Boulder: West-view Press, 2004.

10. Perel' V.I., Eliashberg G.M. Absorption of electromagnetic waves in plasma // Sov. Phys. JETP. -1962. - Vol. 14. - P. 633-637. 